\title{
Bootstrap Goodness-of-Fit Test for the Beta-Binomial Model
}

\author{
S. T. Garren* \\ R. L. Smith ${ }^{\dagger}$ \\ W. W. Piegorsch
}

\begin{abstract}
A common question in the analysis of binary data is how to deal with overdispersion. One widely advocated sampling distribution for overdispersed binary data is the beta-binomial model. For example, this distribution often is used to model litter effects in toxicological experiments. Therein, testing the null hypothesis of a beta-binomial distribution against all other distributions is difficult due to the large variability in litter sizes. We investigate a recent test proposed by Brooks et al. (1997, Biometrics) but find it to have low power in comparison with a Pearson test. Therefore, we generalize the Pearson test statistic by combining Pearson statistics from individual litter sizes, and estimate the $p$-value using bootstrap techniques. A Monte Carlo study confirms the accuracy and power of our omnibus test against a beta-binomial distribution contaminated with a few outliers. The method also is applied to data from environmental toxicity studies.
\end{abstract}

Key words: Beta-binomial; Bootstrap; Goodness-of-fit; Overdispersion; Pearson statistic; Toxicological data.

\footnotetext{
${ }^{*}$ Assistant Professor of Statistics, Division of Statistics, 104 Halsey Hall, University of Virginia, Charlottesville, VA 22903. Research partially supported by NIMH grant MH53259-01A2.

${ }^{\dagger}$ Professor of Statistics, Department of Statistics, University of North Carolina, Chapel Hill, NC 275993260. Research partially supported by NSF grants DMS-9205112 and DMS-9705166.

${ }^{\ddagger}$ Professor of Statistics, Department of Statistics, University of South Carolina, Columbia, SC 29208.
} 


\section{Introduction: Extra-Binomial Variability}

In many experiments encountered in the biological and biomedical sciences, data are generated in the form of proportions, $Y / n$, where $Y$ is a non-negative count and is bounded above by the positive integer $n$. When $n$ is assumed fixed and known, $Y$ might be modeled as binomial $(n, p)$; i.e., view $Y$ as the sum of $n$ independent Bernoulli random variables, $W_{m}$ $(m=1, \ldots, n)$, with $E W_{m}=p$. If some correlation existed among the $W_{m}$, then $Y$ would no longer be distributed as binomial. This situation is not uncommon; e.g., in laboratory tests for developmental toxicity the $W_{m}$ can represent the binary responses of fetuses within a litter of size $n$ from a female rodent exposed to some toxic stimulus (Haseman and Piegorsch, 1994). Since the pregnant rodent is the experimental unit in this situation, the litter-mates represent correlated binary observations, and the sum of those observations may not fit the binomial sampling model. Correlated Bernoulli responses within a litter create what is known as a litter effect and often such an effect is modeled hierarchically. The introduction of heterogeneity in $p$ induces correlation between the $\left\{W_{n}\right\}$ and therefore may be used to model litter effects. If $p$ is beta distributed and $(Y \mid n, p) \sim \operatorname{binomial}(n, p)$, then the marginal density of $Y$ given $n$ is beta-binomial (Williams, 1975; Haseman and Kupper, 1979). Additional details of this model are provided in Section 2.

In cases with strong evidence of extra-binomial variability, the beta-binomial model is preferable to the binomial model. Testing for departure from the binomial distribution has been discussed by Cochran (1954) and Tarone (1979) amongst others. Risko and Margolin (1996) provide a recent review and commentary on these methods. In a study of the statistical features and sources of variability in an assay for heritable mutagenesis (a form of developmental toxicity), Lockhart et al. (1992) questioned whether the beta-binomial was a valid sampling model for overdispersed binomial data they encountered, though their results were inconclusive. This suggests the need for a formal test of whether overdispersion is adequately modeled by a beta-binomial distribution. Several tests have been proposed in the literature, though none of them seem satisfactory for our purposes. We examine in Section 3.2 a recent test proposed by Brooks et al. (1997) but as we will see, the power of the test can be low in some instances

In Section 3.3 a goodness-of-fit test for the beta-binomial model is constructed by boot- 
strapping $\chi^{2}$ tests. Simulation results in Section 4 show that the method provides reasonably accurate estimates of the size of the test, and that the test is powerful against a beta-binomial model contaminated with outliers. Section 5 applies the test to a series of toxicological data sets, and we end in Section 6 with a short discussion.

\section{Description of Beta-Binomial Model}

One characterization of the beta-binomial model employs the following hierarchy: within the context of a developmental toxicity experiment, assume that a given study consists of $J$ litters of animals, and that $n_{i}$ is the number of pups in the $i$ th litter, for $i=1, \ldots, J$. The litter sizes $n_{i}$ are treated as fixed constants. Let $Y_{i}$ denote the number of responses in the $i$ th litter. Conditional on $p_{i}$, the $Y_{i}$ are independent binomial random variables

$$
\left(Y_{i} \mid n_{i}, p_{i}\right) \sim \operatorname{binomial}\left(n_{i}, p_{i}\right), \quad i=1, \ldots, J
$$

The random variables $\left\{p_{i}, i=1, \ldots, J\right\}$ are independent and have the common beta density

$$
f(p \mid \alpha, \beta)=[B(\alpha, \beta)]^{-1} p^{\alpha-1}(1-p)^{\beta-1} \quad(0<p<1)
$$

where $\alpha$ and $\beta$ are unknown positive constants, and $B(\cdot, \cdot)$ is the beta function. The unconditional distribution of $Y_{i}$ is expressed by the beta-binomial probability

$$
P\left(Y_{i}=y \mid n_{i}, \alpha, \beta\right)=\left(\begin{array}{c}
n_{i} \\
y
\end{array}\right) \frac{B\left(\alpha+y, \beta+n_{i}-y\right)}{B(\alpha, \beta)},
$$

for $y=0, \ldots, n_{i} ; i=1, \ldots, J$. If one defines the strictly positive parameters $\mu$ and $\phi$ by

$$
\mu=(\alpha+\beta)^{-1} \alpha \text { and } \phi=(\alpha+\beta)^{-1}
$$

as suggested by Williams (1975), then the mean and variance of $Y_{i}$ can be expressed by

$$
E\left(Y_{i} \mid n_{i}, \mu, \phi\right)=n_{i} \mu \text { and } \operatorname{Var}\left(Y_{i} \mid n_{i}, \mu, \phi\right)=n_{i} \mu(1-\mu)(1+\phi)^{-1}\left(1+n_{i} \phi\right)
$$

for $i=1, \ldots, J$. The parameter $\mu$ may be referred to as the mean parameter of the marginal proportions, where $0<\mu<1$. The parameter $\phi$ is called the dispersion parameter, and if $\phi>0$, then the data are said to be overdispersed. In some settings the variance of $Y_{i}$ may appear smaller than that for the binomial distribution, suggesting underdispersion (Prentice, 
1986; Engel and te Brake, 1993), but this is not common in developmental toxicology and hence we will not study it here.

The maximum likelihood estimator (MLE) of $(\mu, \phi)$ can be shown to be consistent (Lehmann, 1983, pp. 409-413) and is determined numerically. As $\phi \downarrow 0$, the variance of $\left(Y_{i} \mid n_{i}, \mu, \phi\right)$ monotonically decreases to $n_{i} \mu(1-\mu)$, and $\left(Y_{i} \mid n_{i}, \mu, \phi\right)$ converges to a binomial random variable.

\section{Approaches to Goodness-of-Fit Testing}

Suppose the data consist of independent pairs $\left\{\left(Y_{i}, n_{i}\right), i=1, \ldots, J\right\}$ as described in Section 2, and suppose the goal is to test the null hypothesis that the data follow a beta-binomial distribution (1) against the alternative hypothesis that the distribution is not of this form. Throughout, we will use the $(\mu, \phi)$ parametrization (2) to represent a specific member of the beta-binomial family.

\subsection{Previous Test Statistics}

For discrete distributions, Pearson's $\chi^{2}$ statistic is often used for testing goodness-offit. The difficulty with this in the case of toxicity experiments is that the data usually represent information from litters of different sizes, and in this case it is not easy to apply the $\chi^{2}$ test. Mantel and Paul (1987) resolved this problem by assuming that the litter sizes $\left\{n_{i}, i=1, \ldots, J\right\}$ are themselves random variables from some known distribution, and based the Pearson statistic on the unconditional probability distribution of the $Y_{i}$ using the MLE of $(\mu, \phi)$. This approach, however, loses information about the individual litter sizes when determining the observed numbers of the $Y_{i}$, and thus could conceal large variations in the proportions of responses among litters.

A different approach is based on likelihood ratio tests. Pack (1986) proposed using the likelihood ratio for testing the specific question of whether two groups of beta-binomial data have the same or two different values of $(\mu, \phi)$. Lockhart et al. (1992) used the likelihood ratio for testing the beta-binomial model against the alternative that the $Y_{i}$ have independent binomial distributions with parameters $\left(n_{i}, p_{i}\right)$. There are some technical difficulties with this latter approach, however; since the number of unknown parameters $p_{i}$ 
goes to infinity as $J \rightarrow \infty$, the usual asymptotic theory developed for likelihood ratio tests cannot necessarily be applied in this situation.

Liang and McCullagh (1993) proposed a test for determining whether the mean-variance relationship across different litter sizes is consistent with the beta-binomial model. Their method accounted for extra-binomial variability of a form that included the beta-binomial, but employed only a quasi-likelihood fitting algorithm to estimate the model parameters. Thus the formal beta-binomial assumption in (1) was not used. The procedure was presented more as an approach for comparing different extra-binomial variance structures when the mean-variance relationship is the only distributional property under study, rather than as a formal test for goodness-of-fit to the beta-binomial.

More recently, Brooks et al. (1997) considered overdispersion models for developmental toxicity data that included the beta-binomial, but that also allowed for various finite mixtures of binomials and beta-binomials. In fact, the main emphasis of their paper was to employ finite mixture models to determine which model had the best fit, by examining the maximized likelihood function. For assessing the quality of the beta-binomial assumption, they avoided specifying an alternative model by working with the maximized likelihood itself, rather than with a likelihood ratio. Specifically, they first maximized the likelihood of the data under a beta-binomial model, and then simulated from the fitted beta-binomial distribution to determine a null distribution of the maximized likelihood. By implication, they reject the beta-binomial model when the observed test statistic falls in either tail of the null distribution. This produces a two-sided $p$-value, where small $p$-values indicate departure from beta-binomial variability in the original data. For all six of their data examples, this test accepted the null hypothesis of validity for the beta-binomial model as we discuss in Section 5 (with regards to Table 5), but in five of the six cases Brooks et al. (1997) subsequently argued that a better fit could be obtained using some other model. This suggests that their goodness-of-fit test may lack power, so we propose an improved omnibus test in Section 3.3.

In Section 3.2 we demonstrate, by direct calculations in a simple example involving only one litter size, that the test proposed by Brooks et al. (1997) may exhibit low power in comparison with a Pearson $\chi^{2}$ test. This motivates us to return to the Pearson test in 
the general case involving different litter sizes. In Section 3.3 we propose a method for combining Pearson tests for different litter sizes, using bootstrapping to determine the null distribution of the test statistic. The remainder of the paper is concerned with properties and examples of this new test.

\subsection{A Power Comparison}

Consider the following hypothetical situation. The data consist of $J$ litters each of size 3. For given $\lambda \in(0,1)$, a fraction of exactly $\lambda$ of the litters (to the nearest integer) have $y_{i}=0$, and the remainder have $y_{i}=2$. This model is clearly not beta-binomial and any reasonable test ought to reject it for quite small values of $J$.

For each value of $\lambda$, the values of $(\mu, \phi)$ which maximize the log likelihood have been found numerically. For $\lambda \leq 0.25$, the maximum is achieved with $\phi=0$, so the best betabinomial model is binomial in this case. In such a setting, we can compute $f_{0}$ and $f_{1}$, the expected values of the negative log likelihood under both the true model and the best beta-binomial model, respectively, and also $f_{2}$, the variance of the log likelihood for $J=1$ under the best beta-binomial model. For two values of $\lambda, 0.25$ and approximately 0.53 , we find $f_{0}=f_{1}$, so in this case the log likelihood statistic has no power to discriminate between the two models. For other values of $\lambda$, the $\log$ likelihood test will reject the beta-binomial model at approximately the 0.05 significance level if $J>1.96^{2} f_{2} /\left(f_{1}-f_{0}\right)^{2}$. This may be compared with the value of $J$ at which the Pearson statistic would reject the null hypothesis at level 0.05 under the stated data configuration.

Figure 1 shows $f_{0}$ and $f_{1}$ plotted as a function of $\lambda$. It can be seen that the two curves cross twice, but more importantly, they remain close together for most of the range of $\lambda$. In Figure 2, we plot the value of $J$, the sample size for which the beta-binomial model would just be rejected at level 0.05 , for each of the two tests. For nearly all the range of $\lambda$, the Pearson test performs better, and for much of the range, dramatically so.

Although this example covers only one specific data configuration, there is no reason to think that the results are atypical, since the main point is that the expected values of the log likelihood statistic under the null and alternative models may be close to each other even when not exactly equal. 


\subsection{A Bootstrap Test Statistic}

The difficulties of constructing a simple, consistent goodness-of-fit test prompt us to return to the Pearson statistic as in Mantel and Paul (1987), but with some modifications to employ information in the different litter sizes, and to use bootstrapping to determine the null reference distribution. This approach is in line with the general approach to bootstrapping goodness-of-fit statistics advocated by Romano (1988).

Suppose there are $J_{1}$ litters of size $n_{1}, J_{2}$ litters of size $n_{2}$, and so on up to $J_{K}$ litters of size $n_{K}$, where $J_{k}>0(k=1, \ldots, K)$ and $\sum_{k} J_{k}=J$. Our beta-binomial goodness-of-fit test statistic $\tau$ is constructed as follows:

1. Calculate individual Pearson goodness-of-fit test statistics for each litter size. Thus, for each litter size $n=n_{k}(k=1, \ldots, K)$, let $O_{y, n}$ denote the observed number of litters of size $n$ which contain $y$ responses. Similarly, let $E_{y, n}$ denote the expected value under (1) of $O_{y, n}$, assuming $J_{k}$ litters of this size, where $(\mu, \phi)$ is estimated by the MLE $(\hat{\mu}, \hat{\phi})$. Define the individual Pearson statistic to be

$$
Q_{n}=\sum_{y=0}^{n}\left(O_{y, n}-E_{y, n}\right)^{2} / E_{y, n} .
$$

For a particular realization, let $q_{n}$ denote the observed value of $Q_{n}$.

2. Estimate the distribution function of the $q_{n}$ by simulation. Thus, for each litter size $n=n_{k}(k=1, \ldots, K)$, generate a large number $J_{n} M$ of beta-binomial pseudo-random variates with parameters $(\hat{\mu}, \hat{\phi})$, where $M$ does not depend on $n$. Repeat the calculation in step 1 to generate a parametric bootstrap sample $Q_{n, 1}^{*}, \ldots, Q_{n, M}^{*}$ such that each $Q_{n, m}^{*}$ is based on $J_{n}$ litters. Note that $\mu$ and $\phi$ are re-estimated for each bootstrap sample. The distribution function of the $q_{n}$ is estimated to be

$$
\rho_{n}=M^{-1} \sum_{m=1}^{M} I\left(Q_{n, m}^{*}<q_{n}\right),
$$

where $I(\cdot)$ is the indicator function. The null distribution of $\rho_{n}$ is approximately uniform $(0,1)$ though not exactly uniform, even in the limit as $M \rightarrow \infty$, since $Q_{n}$ is discrete.

3. Combine the estimated distribution functions $\rho_{n}$ by computing the estimated $p$-value

$$
\tau=1-\left(\max _{k=1, \ldots, K} \rho_{n_{k}}\right)^{K}
$$


Intuitively, the null hypothesis should be rejected if any $\rho_{n}$ is too large; i.e., if $\tau$ is too small. The power transformation in (3) ensures that, if each $\rho_{n}$ is approximately uniform $(0,1)$, then $\tau$ also is approximately uniform $(0,1)$ so that $\tau$ estimates the $p$-value. Hence, an approximate level $\tau_{0}$ test is obtained by rejecting the null hypothesis whenever $\tau<\tau_{0}$.

Since the distribution of $\tau$ is discrete, our proposed bootstrap method theoretically can be replaced by computation of the exact distribution, but the amount of computing time would be enormous. We prefer, therefore, to use the bootstrap. We explore the operating characteristics of this test in a modest Monte Carlo study in the next section.

\section{Monte Carlo Study}

We performed a Monte Carlo study to determine how well our test statistic proposed in Section 3 performs with a sample size of $J=50$. The accuracy of the test's size was examined by simulating a beta-binomial distribution and testing for departure from it, while the power of the test was examined by simulating a beta-binomial distribution mixed with a binomial distribution and again testing for departure from the beta-binomial. Both sets of simulations used litter sizes from a toxicological study considered below, in which the 50 litters ranged in size from 6 to 18 (see Table 1a). To study size and power characteristics, the underlying parameters of the beta-binomial distribution were chosen to vary over all combinations of $\mu \in\{0.05,0.1,0.15\}$ and $\phi \in\{0,0.05,0.1\}$. In the alternative distribution for the power study the binomial distribution was assigned the response probability $p \in\{0.7,0.8,0.9\}$, and the beta-binomial distribution was chosen with mixing probability $\{0.85,0.9,0.95\}$. Notice that when the alternative model has a large mixing probability, the model can be viewed as a beta-binomial model contaminated with a few outliers. The number of bootstrap samples was set to $M=1000$, and the number of independent replications was 2000 . The nominal levels, $\tau_{0}$, were taken as $0.1,0.05,0.025$, and 0.01 . The estimated rejection probabilities (size or power) are the proportions of the 2000 replicates where $\tau<\tau_{0}$. These appear in Tables $2 \mathrm{a}$ (size) and 3a (power). With 2000 replicates, our estimates of the

rejection probability, $\gamma$, have approximate standard errors of $\sqrt{\gamma(1-\gamma) / 2000}$. For example, these estimates of standard error are $0.0067,0.0049,0.0035$, and 0.0022 when $\gamma$ has values 
$0.1,0.05,0.025$, and 0.01 , respectively.

Table 2a illustrates that the estimated size is somewhat close to its nominal level, $\tau_{0}$, although the tendency is to be slightly above it. Table 3a tends to suggest that large mixing probabilities produce large power when the overall mean, $\mu$, of the beta-binomial model differs greatly from the response probability, $p$, in the binomial model. Large mixing probabilities $(\simeq 0.95)$ frequently produce at least one extreme value of $y$, which increases the corresponding statistics $O_{y, n}$ and $\rho_{n}$ and decreases $\tau$, since the $E_{y, n}$ typically are not greatly influenced by a small number of extreme values of $y$.

To explore further the operating characteristics of this bootstrap approach, we increased the number of litters to $J=100$ in the Monte Carlo evaluations. Tables $2 \mathrm{~b}$ and $3 \mathrm{~b}$ were produced, respectively, in the same way as Tables $2 \mathrm{a}$ and $3 \mathrm{a}$, except Tables $2 \mathrm{~b}$ and $3 \mathrm{~b}$ used $J=100$ litters instead of 50 . These additional litters were generated by doubling the 50

litter size frequencies, $J_{k}$, in Table 1a. Results from Tables $2 \mathrm{a}$ and $2 \mathrm{~b}$ are similar, indicating that perhaps some of the error when estimating size comes from using a limited number of bootstrap samples and replications, rather than from using only 50 or 100 litters. Table $3 \mathrm{~b}$ shows greater power than Table 3a for the binomial probability $p=0.9$. The other values of $p$ did not result in much difference in power between Tables $3 \mathrm{a}$ and $3 \mathrm{~b}$.

\section{$5 \quad$ Examples}

As an illustration we applied our goodness-of-fit test statistic to data from four experiments involving pregnant mice, studied originally by Lockhart et al. (1992). Those experiments involved matings between a male and a female mouse to examine damage in the resulting embryos based on dominant lethal mutations. To assess such damage, approximately two weeks after mating, the pregnant females were sacrificed and their uterine contents were examined. For each litter the number of viable implants and the number of non-viable implants were determined, where viable was defined before the experiment begins (Lockhart et al., 1991). None of these parent mice were exposed to any toxic chemicals before or during the experiment.

Lockhart et al. (1992) noted that the majority of proportions from these studies ex- 
hibited significant departure from the simple binomial model, and considered use of the beta-binomial in (1) to model the overdispersion. A concern of interest was whether this was an adequate assumption, i.e., was there adequate goodness-of-fit for the beta-binomial model? To answer this question, we can apply the bootstrap method from Section 4. Our goodness-of-fit results are summarized in Table 4, which are based on the data from Tables 1a-1d.

In Table 4, the number of bootstrap samples used is $M=100,000$ when determining the observed significance level, $\tau$. The table indicates that data sets in Tables 1c and $1 \mathrm{~d}$ are significant at level 0.05. A more detailed examination of the data set in Table $1 \mathrm{~d}$ indicated the presence of three unlikely $(y, n)$ pairs: $(7,7),(9,9)$, and $(5,8)$. These appear to be outliers since they occur with very small probabilities under the associated MLE $(\hat{\mu}, \hat{\phi})=(0.068,0.064)$. When these three data pairs were removed, the $p$-value increased from $\tau=0.000$ to $\tau=0.054$. This shows that although the outliers partly explain the failure of the beta-binomial distribution, they are not the sole reason for it. Even when the outliers are removed, the test is borderline significant.

We also analyzed six of the developmental toxicity data sets analyzed by Brooks et al. (1997), again using $M=100,000$ bootstrap samples. We summarize the results in Table 5 . We determined $p$-values, $\tau$, based on our procedure described in Section 3.3, and we also determined $p$-values based on the procedure proposed by Brooks et al. (1997). The first two data sets were published by Brooks et al. (1997). Data sets \#3-5 were originally published by Haseman and Soares (1976), and the sixth was originally published by Aeschbacher et al. (1977). [The tables in Brooks et al. (1997) contain some minor typographical errors: in Table 1 the entry at position $(8,14)$ should be moved to $(9,14)$; in Table 2 the entry of "one" should appear at position $(11,16)$; in Table 4 the entry at position $(9,10)$ should be moved to $(10,10)$.]

Brooks et al. (1997) stated that for data set \#1 in (our) Table 5, their log likelihood test statistic ranked 45 among 99 simulated values; i.e., their simulated $p$-value for testing departure from the beta-binomial was approximately 0.90 since their test is two-tailed. Using their test statistic we simulated their $p$-value to be 0.929 , which is relatively close to 0.90. They also noted that for all of the data sets in Table 5 the beta-binomial appeared to 
provide an adequate fit. Our simulated $p$-values of their test statistic in Table 5 are 0.929 , $0.884,0.796,0.844,0.577$, and 0.923 for the data sets \#1-6, respectively, and thus confirm their findings.

As shown in our Table 5, however, our bootstrap test statistic produced $p$-values, $\tau$, of $0.144,0.231,0.000,0.000,0.009$, and 0.375 for the data sets \#1-6, respectively. We therefore conclude that data sets \#3-5 appear to depart significantly from the beta-binomial model. The discrepancies between our $p$-values $(\tau)$ and their $p$-values might be explained by the lack of power the simulated likelihood comparison can experience, as we noted in Section 3.2.

One comparison made by Brooks et al. (1997) was between a null model of beta-binomial and an alternative of a discrete mixture of binomials. Because these models are not nested, they required simulation to evaluate the $p$-value of the likelihood ratio test statistic. For data set \#1 they rejected the beta-binomial but with a moderate $p$-value (.04). For data sets \#2 and \#4 they stated that the comparison preferred the mixture of binomials, but they did not quote a $p$-value. For \#3 they preferred the beta-binomial while in the case of \#5 and \#6 their test did not indicate any preference between the two models.

In a further comparison, Brooks et al. (1997) also compared the beta-binomial as null hypothesis with a mixture of one beta-binomial component and one binomial component as the alternative. Although these models are nested, it is still necessary to use simulation because of non-regular properties of the test statistic. In this case, the results in their Table 10 showed very strong evidence against the beta-binomial null hypothesis in the case of data sets \#4 and \#5, and rather weaker evidence against the beta-binomial in the case of each of the others except \#6. In each of \#1, \#2, \#3 they rejected the null hypothesis based on 100 simulations, but the actual likelihood ratio test statistics in the data are not very much greater than the values in the simulations.

Our own results, shown in Table 5, show that the beta-binomial model is accepted by our test in the case of data sets \#1, \#2 and \#6, but in the other three cases, it is decisively rejected. In the case of $\# 3$, the evidence we find against the beta-binomial model, as judged by the $p$-value, seems even stronger than that found by Brooks et al. (1997), which could possibly indicate that some other alternative model would fit better than any of the alternatives considered so far. It therefore appears that the two methods are in agreement in 
those cases which are clear-cut, but in the other cases, neither procedure clearly dominates the other, and we believe there are good reasons for considering the bootstrap test instead of or in addition to the range of tests against specific alternatives advocated by Brooks et al. (1997).

\section{Discussion}

The beta-binomial model is applied frequently when analyzing proportion data with some form of litter effect. This model is quite rich, has some intuitive appeal, and is relatively simple to use since its probability distribution is tractable. As noted in Section 3, however, standard goodness-of-fit approaches are difficult to employ. Our method is an attempt at determining simple significance levels for testing fit to the beta-binomial model, and the test seems to be powerful when the data are generated by certain mixture models. In particular, our method is powerful when the alternative model is beta-binomial contaminated with a small proportion of outliers.

Our test statistic, $\tau$, can be extended easily to other models when testing goodnessof-fit. For example, one may wish to model toxicity data by a beta-binomial distribution, where the population mean, $\mu$, is a function of the dose of a chemical given to the dam (Catalano and Ryan, 1994, Sec. 4). Additional parameters may need to be estimated by maximum likelihood or some other approach, although the basic technique for computing $\tau$, the observed significance level, remains the same. Likewise, goodness-of-fit to other forms of extra-binomial model such as the correlated-binomial model or the beta-correlated-binomial model (Brooks et al., 1997) may be tested using this same basic technique. Since $\tau$ does not require specification of an alternative model, our test procedure maintains a similar simplicity to that of Pearson's goodness-of-fit test, so we have only one $p$-value to simulate for each data set. Brooks et al. (1997), on the other hand, employed a sequence of likelihood ratio tests against different alternative models, and thus required a different Monte Carlo $p$-value for each alternative model.

When a specific alternative model is given, then the likelihood ratio test against that alternative, as advocated by Brooks et al. (1997), is likely to be more powerful than 
any general-purpose test such as ours. However, it is not always clear what is the right alternative model to test, and in that situation we believe our procedure to be a fully competitive alternative.

We note in closing that a sample of 50 litters may appear large, encouraging use of the $\chi^{2}$ approximation of $Q_{n}$, the individual Pearson statistics, rather than the bootstrap. But in fact, a sample of 50 litters is quite small, relative to asymptotic approximations. For example, if $(\mu, \phi)=(0.05,0)$ and $J=50$, then $E_{y, n}$ is much less than 1 for most values of $(y, n)$. Thus, in most practical situations $J=50$ may not be a large enough number of litters to validate replacing the bootstrap with some asymptotic approximation.

\section{Acknowledgements}

Special thanks are due to Drs. Beth Gladen and David Umbach for their helpful suggestions. We also thank Dr. Steve Brooks for verifying that there were some typographical errors in the tables of Brooks et al. (1997), and also for confirming that a test proposed there was intended to be two-sided.

\section{References}

[1] Aeschbacher, H. U., Vuataz, L., Sotek, J., and Stalder, R. (1977), "The use of the beta-binomial distribution in dominant-lethal testing for 'weak mutagenic activity' (Part 1)," Mutation Research 44, 369-390.

[2] Brooks, S. P., Morgan, B. J. T., Ridout, M. S., and Pack, S. E. (1997), "Finite Mixture Models for Proportions," Biometrics 53, 1097-1115.

[3] Catalano, P. J. and Ryan, L. M. (1994), "Statistical methods in developmental toxicology," in Handbook of Statistics Volume 12: Environmental Statistics, eds. G. P. Patil and C. R. Rao, New York: North-Holland/Elsevier, pp. 507-534.

[4] Cochran, W. G. (1954), "Some methods for strengthening the common $\chi^{2}$ tests," Biometrics 10, 417-451. 
[5] Engel, B. and te Brake, J. (1993), "Analysis of embryonic development with a model for underdispersion or overdispersion relative to binomial variation," Biometrics 49, $269-279$.

[6] Haseman, J. K. and Kupper, L. L. (1979), "Analysis of dichotomous response data from certain toxicological experiments," Biometrics 35, 281-293.

[7] Haseman, J. K. and Piegorsch, W. W. (1994), "Statistical analysis of developmental toxicity data" in Devel. Toxic., eds. C. Kimmel and J. Buelke-Sam, New York: Raven Press, pp. 349-361.

[8] Haseman, J. K. and Soares, E. R. (1976), "The distribution of fetal death in control mice and its implications on statistical tests for dominant lethal effects," Mutation Research 41, 277-288.

[9] Lehmann, E. L. (1983), Theory of Point Estimation, New York: John Wiley.

[10] Liang, K.-Y. and McCullagh, P. (1993), "Case studies in binary dispersion," Biometrics 49, 623-630.

[11] Lockhart, A.-M., Bishop, J. B., and Piegorsch, W. W. (1991), "Issues regarding data acquisition and analysis in the dominant lethal assay," Proc. Amer. Statist. Assoc., Biopharm. Sect., 234-237.

[12] Lockhart, A.-M., Piegorsch, W. W., and Bishop, J. B. (1992), "Assessing overdispersion and dose response in the male dominant lethal assay," Mutation Res. 272, 35-58.

[13] Mantel, N. and Paul, S. R. (1987), "Goodness-of-fit issues in toxicological experiments involving litters of varying size" in Biostatistics, eds. I. B. MacNeill and G. J. Umphrey, New York: Reidel Publishing Company, pp. 169-176.

[14] Pack, S. E. (1986), "Hypothesis testing for proportions with overdispersion," Biometrics 42, 967-972.

[15] Prentice, R. L. (1986), "Binary regression using an extended beta-binomial distribution, with discussion of correlation induced by covariate measurement errors," J. Amer. Statist. Assoc. 81, 321-327. 
[16] Risko, K. J. and Margolin, B. H. (1996), "Some observations on detecting extrabinomial variability within the beta-binomial model" in Statistics in Toxicology, ed. B. J. T. Morgan, Oxford: Clarendon Press, pp. 57-65.

[17] Romano, J. P. (1988), "A bootstrap revival of some nonparametric distance tests," $J$. Amer. Statist. Assoc. 83, 698-708.

[18] Tarone, R. E. (1979), "Testing the goodness-of-fit of the binomial distribution," Biometrika 66, 585-590.

[19] Williams, D. A. (1975), "The analysis of binary responses from toxicological experiments involving reproduction and teratogenicity," Biometrics, 31, 949-952. 


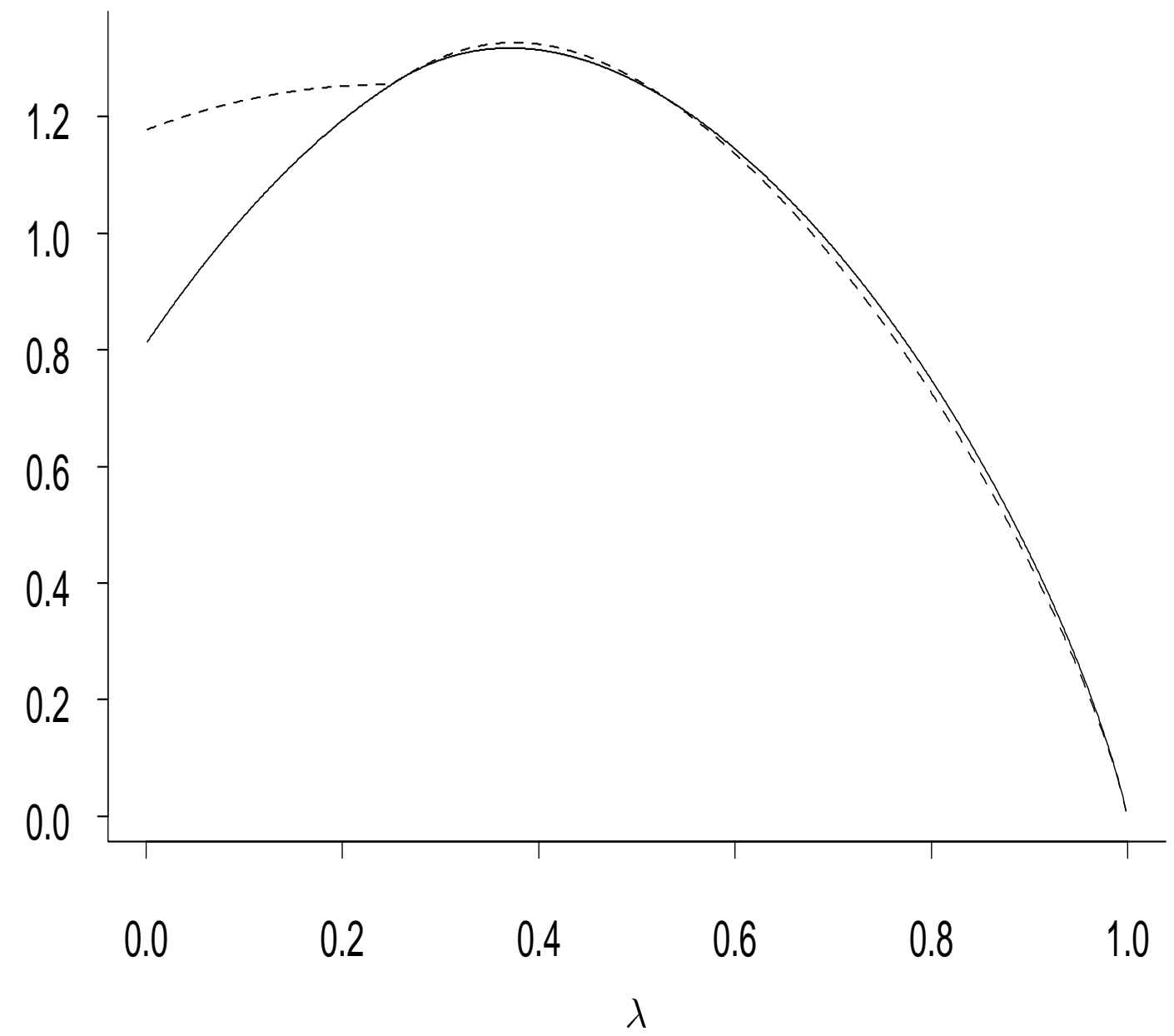

Figure 1. The mean values of the negative log likelihood test statistic computed under the beta-binomial model when the beta-binomial model is true $\left(f_{1}\right.$, dashed curve) and under the model described in the text $\left(f_{0}\right.$, solid curve). The two curves cross at $\lambda=0.25$ and $\lambda=0.53$, and are very close throughout the range $0.25<\lambda<1$. 


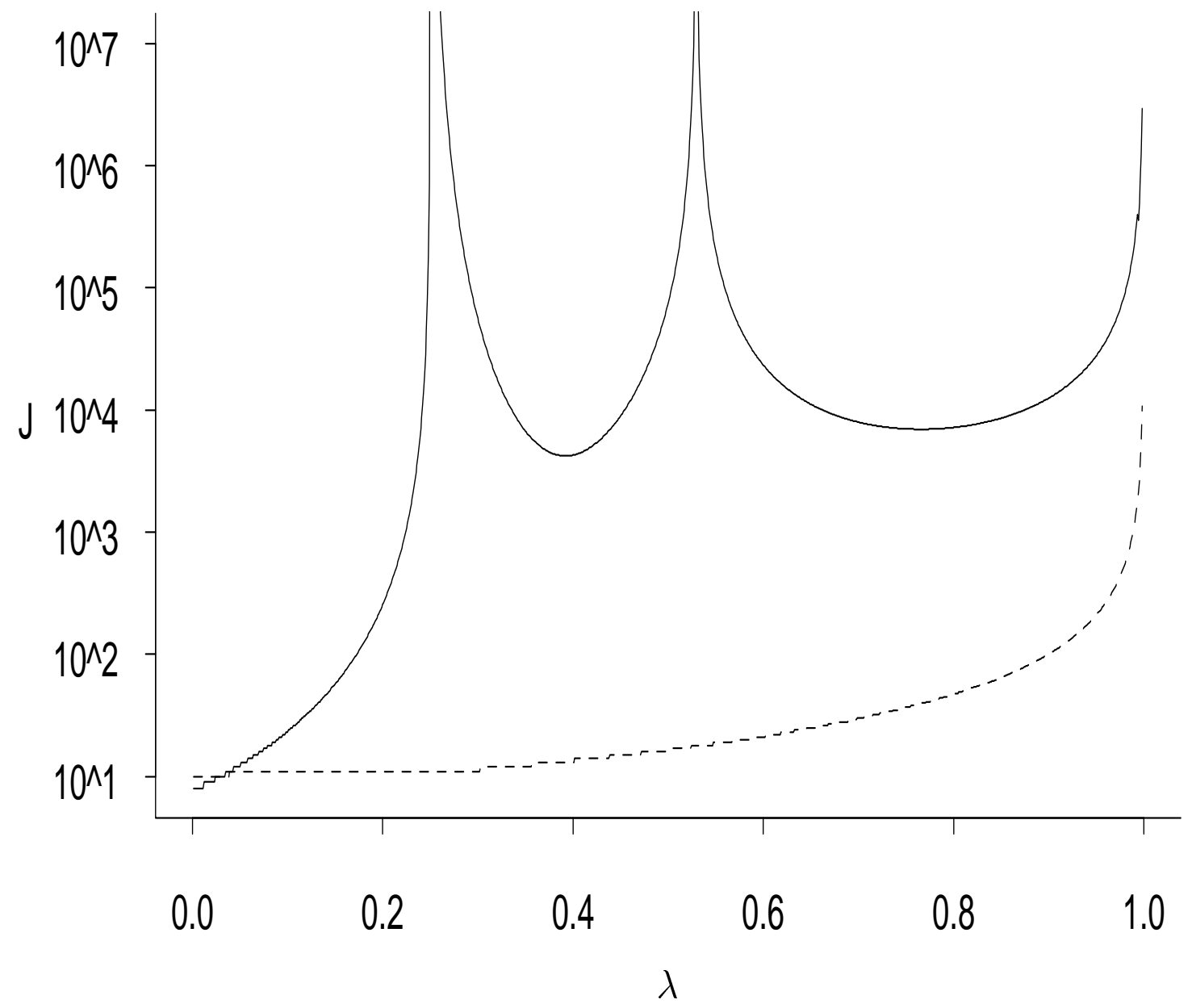

Figure 2. Sample size needed to reject the beta-binomial model when the alternative model is true, assuming a significance level 0.05. Solid curve: Log likelihood test. Dashed curve: Pearson $\chi^{2}$ test. 
TABLE 1a. Frequency of litter sizes with number of non-viable implants, from Lockhart et al. (1992).

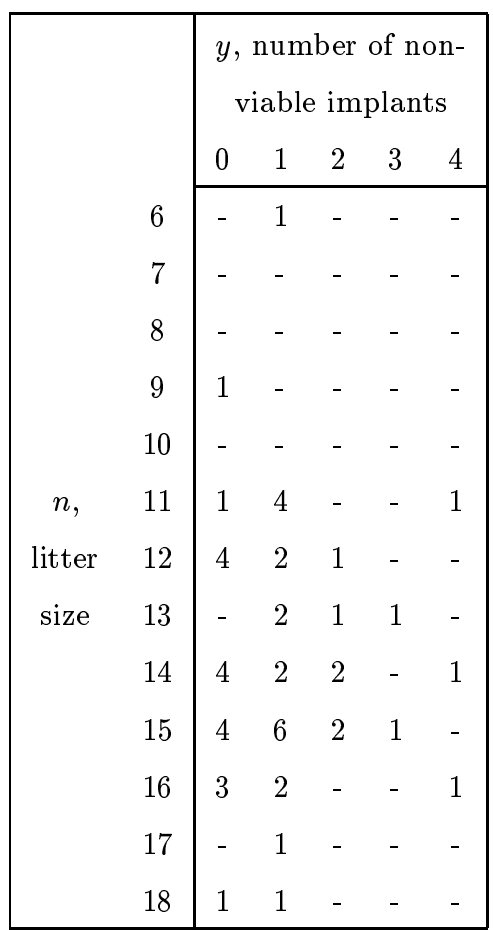

Note: This data set consists of $J=50$ litters.

TABLE 1b. Frequency of litter sizes with number of non-viable implants, from Lockhart et al. (1992).

\begin{tabular}{|c|c|c|c|c|}
\hline & & $\begin{array}{c}y, \text { nun } \\
\text { viabl }\end{array}$ & per & $\begin{array}{l}\text { f non- } \\
\text { lants }\end{array}$ \\
\hline & & 0 & 1 & 2 \\
\hline & 7 & 2 & - & - \\
\hline & 8 & 3 & 2 & - \\
\hline & 9 & 13 & 6 & 2 \\
\hline$n$ & 10 & 8 & 4 & - \\
\hline litter & 11 & 5 & 2 & 1 \\
\hline size & 12 & 2 & 1 & - \\
\hline & 13 & 3 & - & 1 \\
\hline & 14 & - & 1 & - \\
\hline & 15 & 1 & - & - \\
\hline
\end{tabular}

Note: This data set consists of $J=57$ litters. 
TABLE 1c. Frequency of litter sizes with number of non-viable implants, from Lockhart et al. (1992).

\begin{tabular}{|c|c|c|c|c|c|c|}
\hline & & & able & $\mathrm{m}]$ & an & \\
\hline & & 0 & 1 & 2 & 3 & 4 \\
\hline & 2 & 1 & - & - & & \\
\hline & 3 & 2 & 1 & - & - & \\
\hline & 4 & - & - & - & 1 & - \\
\hline & 5 & 2 & - & - & - & - \\
\hline & 6 & - & 1 & - & - & - \\
\hline & 7 & 3 & 2 & - & - & - \\
\hline$n$ & 8 & 3 & - & - & 1 & - \\
\hline litter & 9 & 9 & 3 & - & - & - \\
\hline size & 10 & 20 & 9 & 1 & 1 & - \\
\hline & 11 & 42 & 15 & 4 & - & - \\
\hline & 12 & 26 & 15 & 4 & - & - \\
\hline & 13 & 10 & 5 & 4 & - & 2 \\
\hline & 14 & 5 & 2 & 2 & - & 1 \\
\hline & 15 & - & 1 & - & - & 2 \\
\hline & 16 & 1 & - & - & - & - \\
\hline
\end{tabular}

Note: This data set consists of $J=201$ litters. 
TABLE 1d. Frequency of litter sizes with number of non-viable implants, from Lockhart et al. (1992).

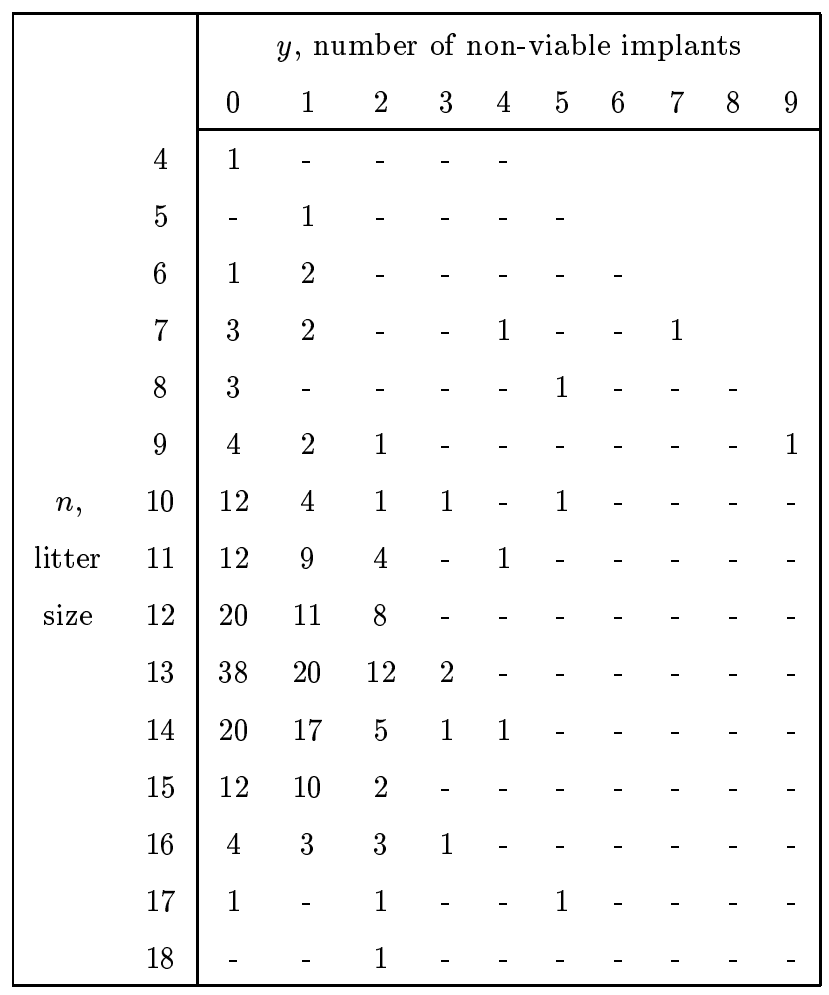

Note: This data set consists of $J=263$ litters. 
TABLE 2a. Estimated sizes of proposed test, based on simulations from beta-binomial distributions using 50 litters.

\begin{tabular}{|c|c||c|c|c|c|}
\hline \multicolumn{2}{|c||}{ beta-binomial } & \multicolumn{4}{c|}{ Estimated size at nominal level $\tau_{0}$} \\
\hline$\mu$ & $\phi$ & $\tau_{0}=0.1$ & $\tau_{0}=0.05$ & $\tau_{0}=0.025$ & $\tau_{0}=0.01$ \\
\hline 0.05 & 0.00 & 0.0765 & 0.0420 & 0.0180 & 0.0060 \\
0.05 & 0.05 & 0.1155 & 0.0675 & 0.0305 & 0.0210 \\
0.05 & 0.10 & 0.1225 & 0.0710 & 0.0395 & 0.0250 \\
0.10 & 0.00 & 0.0855 & 0.0450 & 0.0265 & 0.0160 \\
0.10 & 0.05 & 0.1100 & 0.0655 & 0.0330 & 0.0195 \\
0.10 & 0.10 & 0.1180 & 0.0635 & 0.0260 & 0.0190 \\
0.15 & 0.00 & 0.0835 & 0.0465 & 0.0230 & 0.0155 \\
0.15 & 0.05 & 0.1165 & 0.0625 & 0.0285 & 0.0160 \\
0.15 & 0.10 & 0.1025 & 0.0585 & 0.0300 & 0.0210 \\
\hline
\end{tabular}

TABLE 2b. Estimated sizes of proposed test, based on simulations from beta-binomial distributions using 100 litters.

\begin{tabular}{|c|c||c|c|c|c|}
\hline \multicolumn{2}{|c||}{ beta-binomial } & \multicolumn{4}{c|}{ Estimated size at nominal level $\tau_{0}$} \\
\hline$\mu$ & $\phi$ & $\tau_{0}=0.1$ & $\tau_{0}=0.05$ & $\tau_{0}=0.025$ & $\tau_{0}=0.01$ \\
\hline 0.05 & 0.00 & 0.0775 & 0.0365 & 0.0185 & 0.0125 \\
0.05 & 0.05 & 0.1270 & 0.0770 & 0.0395 & 0.0250 \\
0.05 & 0.10 & 0.1155 & 0.0615 & 0.0295 & 0.0195 \\
0.10 & 0.00 & 0.0925 & 0.0505 & 0.0285 & 0.0180 \\
0.10 & 0.05 & 0.0980 & 0.0570 & 0.0295 & 0.0220 \\
0.10 & 0.10 & 0.1170 & 0.0600 & 0.0280 & 0.0190 \\
0.15 & 0.00 & 0.0860 & 0.0500 & 0.0255 & 0.0160 \\
0.15 & 0.05 & 0.1110 & 0.0610 & 0.0335 & 0.0275 \\
0.15 & 0.10 & 0.0985 & 0.0605 & 0.0310 & 0.0220 \\
\hline
\end{tabular}


TABLE 3a. Estimated power of proposed test, based on simulations from mixture models using 50 litters.

\begin{tabular}{|c|c|c|c|c|c|c|c|}
\hline \multicolumn{2}{|c|}{ beta-binomial } & \multirow{2}{*}{$\frac{\text { binomial }}{p}$} & \multirow{2}{*}{$\begin{array}{c}\text { mixing } \\
\text { probability }\end{array}$} & \multicolumn{4}{|c|}{ Estimated power at nominal level $\tau_{0}$} \\
\hline$\mu$ & $\phi$ & & & $\tau_{0}=0.1$ & $\tau_{0}=0.05$ & $\tau_{0}=0.025$ & $\tau_{0}=0.01$ \\
\hline 0.05 & 0.00 & 0.7 & 0.85 & 0.2530 & 0.1495 & 0.0785 & 0.0545 \\
\hline 0.05 & 0.05 & 0.7 & 0.90 & 0.2510 & 0.1445 & 0.0790 & 0.0595 \\
\hline 0.05 & 0.10 & 0.7 & 0.95 & 0.3475 & 0.2420 & 0.1470 & 0.1070 \\
\hline 0.10 & 0.00 & 0.8 & 0.85 & 0.4200 & 0.2550 & 0.1465 & 0.1115 \\
\hline 0.10 & 0.05 & 0.8 & 0.90 & 0.4635 & 0.3185 & 0.1990 & 0.1360 \\
\hline 0.10 & 0.10 & 0.8 & 0.95 & 0.5215 & 0.3845 & 0.2500 & 0.1875 \\
\hline 0.15 & 0.00 & 0.9 & 0.85 & 0.5465 & 0.3915 & 0.2555 & 0.1970 \\
\hline 0.15 & 0.05 & 0.9 & 0.90 & 0.5720 & 0.4355 & 0.2855 & 0.2225 \\
\hline 0.15 & 0.10 & 0.9 & 0.95 & 0.6600 & 0.5295 & 0.3675 & 0.2910 \\
\hline
\end{tabular}

TABLE 3b. Estimated power of proposed test, based on simulations from mixture models using 100 litters.

\begin{tabular}{|c|c|c|c|c|c|c|c|}
\hline \multicolumn{2}{|c|}{ beta-binomial } & \multirow{2}{*}{$\frac{\text { binomial }}{p}$} & \multirow{2}{*}{$\begin{array}{c}\text { mixing } \\
\text { probability }\end{array}$} & \multicolumn{4}{|c|}{ Estimated power at nominal level $\tau_{0}$} \\
\hline$\mu$ & $\phi$ & & & $\tau_{0}=0.1$ & $\tau_{0}=0.05$ & $\tau_{0}=0.025$ & $\tau_{0}=0.01$ \\
\hline 0.05 & 0.00 & 0.7 & 0.85 & 0.2850 & 0.1410 & 0.0705 & 0.0445 \\
\hline 0.05 & 0.05 & 0.7 & 0.90 & 0.2370 & 0.1300 & 0.0605 & 0.0430 \\
\hline 0.05 & 0.10 & 0.7 & 0.95 & 0.3195 & 0.1960 & 0.1145 & 0.0725 \\
\hline 0.10 & 0.00 & 0.8 & 0.85 & 0.5735 & 0.3850 & 0.2005 & 0.1340 \\
\hline 0.10 & 0.05 & 0.8 & 0.90 & 0.4880 & 0.3070 & 0.1685 & 0.1065 \\
\hline 0.10 & 0.10 & 0.8 & 0.95 & 0.5685 & 0.4025 & 0.2475 & 0.1775 \\
\hline 0.15 & 0.00 & 0.9 & 0.85 & 0.8050 & 0.6615 & 0.4825 & 0.3800 \\
\hline 0.15 & 0.05 & 0.9 & 0.90 & 0.7045 & 0.5700 & 0.3975 & 0.3040 \\
\hline 0.15 & 0.10 & 0.9 & 0.95 & 0.7850 & 0.6350 & 0.4500 & 0.3520 \\
\hline
\end{tabular}


TABLE 4. Analysis of dominant lethal data.

\begin{tabular}{|c|c|c|c|c|}
\hline \multirow[b]{2}{*}{ Table \# } & \multirow{2}{*}{$\begin{array}{l}\text { Number } \\
\text { of litters }\end{array}$} & \multicolumn{2}{|c|}{ MLE } & \multirow{2}{*}{$\begin{array}{c}p \text {-value } \\
\tau\end{array}$} \\
\hline & & $\hat{\mu}$ & $\hat{\phi}$ & \\
\hline $1 \mathrm{a}$ & 50 & 0.075 & 0.021 & 0.333 \\
\hline $1 b$ & 57 & 0.037 & 0.000 & 0.904 \\
\hline $1 c$ & 201 & 0.051 & 0.041 & 0.010 \\
\hline $1 d$ & 263 & 0.068 & 0.064 & 0.000 \\
\hline
\end{tabular}

TABLE 5. Analysis of data sets in Brooks et al. (1997).

\begin{tabular}{|c|c||c|c||c|c|}
\hline \multirow{2}{*}{$\begin{array}{c}\text { Data set } \\
\text { number }\end{array}$} & \multirow{2}{*}{$\begin{array}{c}\text { Number } \\
\text { of litters }\end{array}$} & \multicolumn{2}{c||}{ MLE } & \multicolumn{2}{c|}{$p$-value } \\
\cline { 3 - 6 } & 205 & 0.090 & 0.074 & 0.144 & 0.929 \\
2 & 211 & 0.112 & 0.111 & 0.231 & 0.884 \\
3 & 524 & 0.090 & 0.073 & 0.000 & 0.796 \\
4 & 1328 & 0.109 & 0.045 & 0.000 & 0.844 \\
5 & 554 & 0.074 & 0.081 & 0.009 & 0.577 \\
6 & 127 & 0.069 & 0.063 & 0.375 & 0.923 \\
\hline
\end{tabular}

Note: We simulated the results in the far right column using the procedure proposed by Brooks et al., and we simulated $\tau$ using our procedure. 